\title{
Flora nativa comercializada como recurso medicinal em Parnaíba, Piauí, Nordeste do Brasil
}

\author{
Maria Hortencia Borges dos Santos ${ }^{1 \star}$ (D), Ethyênne Moraes Bastos ${ }^{1}$ (D), Juliana Cardozo de \\ Farias $^{1}$ (D), Irlaine Rodrigues Vieira ${ }^{2}$ (D), Roseli Farias Melo de Barros $^{3}$ (D) \\ 1 Mestrado em Desenvolvimento e Meio Ambiente - PRODEMA, Campus Universitário Ministro Petrônio Portella - \\ Ininga, Teresina - PI, 64049-550. \\ 2 Doutorado em Desenvolvimento e Meio Ambiente - PRODEMA, Campus Universitário Ministro Petrônio Portella \\ - Ininga, Teresina - PI, 64049-550. \\ 3 Departamento de Biologia, Mestrado e Doutorado em Desenvolvimento e Meio Ambiente da Universidade Federal \\ do Piauí - UFPI, Campus Universitário Ministro Petrônio Portella - Ininga, Teresina - PI, 64049-550. \\ * Autor para correspondência: mariabioflor@hotmail.com.
}

Recebido em 24 de dezembro de 2019.

Aceito em 06 de janeiro de 2021.

Publicado em 15 de abril de 2021.

Resumo - As plantas nativas comercializadas em mercados públicos são empregadas como alimentícias, ritualísticas e medicinais, colaborando na renda mensal de erveiros. No estado do Piauí, a comercialização de plantas representa fonte de renda e um importante recurso no tratamento de enfermidades pela população. Objetivou-se identificar como e quais plantas medicinais estão sendo comercializadas nesse estado. Foram aplicados formulários semiestruturados aos 34 vendedores de plantas medicinais dos mercados públicos de Parnaíba, Piauí. As plantas foram coletadas e incorporadas ao Herbário Graziela Barroso (TEPB) da Universidade Federal do Piauí. Utilizou-se o Fator de Consenso do Informante, Importância Relativa e o Índice de Similaridade de Jaccard para análise dos dados. Registrou-se 46 espécies nativas. A família mais representativa foi Fabaceae e a parte mais comercializada foi a casca. As plantas arbóreas foram predominantes e os chás destacaram-se. O sistema corporal mais citado foi Sinais e sintomas em gerais. A espécie Myracrodruon urundeuva Allemão foi a mais citada. A comercialização de plantas nativas nos mercados de Parnaíba é realizada por erveiros. Diversas espécies são empregadas no tratamento de doenças. Algumas plantas nativas medicinais estão em situação de risco. Deste modo, são necessários estudos futuros avaliando o impacto da coleta e de como esse comércio impacta a flora regional.

Palavras-chave: Conhecimento tradicional. Etnobotânica urbana. Mercados públicos.

\section{Native flora marketed as a medicinal resource in Parnaíba, Piauí}

\begin{abstract}
Native plants sold in public markets are used as foodstuffs, as well as ritualistic and medicinal products, contributing to the monthly income of herbalists. In the state of Piauí, the sale of plants represents a source of income and an important resource in the treatment of diseases by the population. The objective was to identify how and which medicinal plants are being commercialized in this state. Semi-structured forms were applied to the 34 sellers of medicinal plants in the public markets of Parnaíba, Piauí. The plants were collected and incorporated into the Herbarium Graziela Barroso (TEPB) of the Federal University of Piauí. The Informant Consensus Factor, Relative Importance and the Jaccard Similarity Index were used for
\end{abstract}


data analysis. 46 native species were registered. The most representative family was Fabaceae and the most commercialized part was the bark. Tree plants were predominant and the teas stood out. The most cited body system was Signs and symptoms in general. The species Myracrodruon urundeuva (Allemão) was the most cited. The sale of native plants in the Parnaíba markets is carried out by herbalists. Various species are used to treat diseases. Some native medicinal plants are at risk. Thus, future studies are needed to assess the impact of collection and how this trade impacts regional flora.

Keywords: Traditional knowledge. Urban ethnobotany. Public markets.

\section{Flora nativa comercializada como recurso medicinal en Parnaíba, Piauí}

Resumen - Las plantas nativas vendidas en los mercados públicos se utilizan como alimento, así como también productos rituales y medicinales, contribuyendo al ingreso mensual de los herboristas. En el estado de Piauí, la venta de plantas representa una fuente de ingresos y un recurso importante en el tratamiento de enfermedades por parte de la población. El objetivo fue identificar cómo y qué plantas medicinales se comercializan en este estado. Se aplicaron formularios semiestructuradas a los 34 vendedores de plantas medicinales en los mercados públicos de Parnaíba, Piauí. Las plantas fueron recolectadas e incorporadas al Herbario Graziela Barroso (TEPB) de la Universidad Federal de Piauí. El factor de consenso del informante, la importancia relativa y el índice de similitud de Jaccard se utilizaron para el análisis de datos. Se registraron 46 especies nativas. La familia más representativa fue Fabaceae y la parte más comercializada fue la corteza. Predominaron las plantas arbóreas y se destacaron los tés. El sistema corporal más citado fue Signos y síntomas en general. La especie Myracrodruon urundeuva (Allemão) fue la más citada. La venta de plantas autóctonas en los mercados de Parnaíba la realizan los herboristas. Varias especies se utilizan para tratar enfermedades. Algunas plantas medicinales nativas están en riesgo. Por lo tanto, se necesitan más estudios para evaluar el impacto de la recolección y cómo este comercio afecta la flora regional.

Palabras-clave: Conocimientos tradicionales. Etnobotánica urbana. Mercados públicos.

\section{Introdução}

De acordo com a história, o homem explorou a natureza buscando solucionar as enfermidades que o afligia, por meio de plantas e animais, além disso, também as empregou para se alimentar, construir abrigos e roupas (Freitas et al. 2012). Atualmente, a humanidade faz uso de uma ampla variedade de espécies vegetais, explorando-as com distintas finalidades, dentre as quais, destacamse o uso na alimentação e a cura de doenças (Silva 2012). Desta forma, o uso de inúmeras espécies terapêuticas, têm sido amplamente disseminadas em comunidade rurais e urbanas do país (Roque et al. 2010). Estima-se que cerca de $80 \%$ da população mundial necessita da medicina tradicional, principalmente em áreas distantes nos países em desenvolvimento, onde a única fonte disponível de cuidado com a saúde é a utilização de plantas (Hoang e Tran 2019).

Mercados são locais que possibilitam evidenciar aspectos importantes sobre a utilização da flora e fauna nativa ou exótica de uma região (Almeida e Albuquerque 2002). Além disso, expõem a variedade de espécies úteis e podem auxiliar na elaboração de planos de conservação dos recursos naturais (Albuquerque et al. 2007). Neste âmbito, muitos pesquisadores têm se dedicado ao estudo 
da comercialização de plantas em feiras e/ou mercados (Monteiro et al. 2010; Oliveira et al. 2010; Conceição et al. 2011; Lima et al. 2011; Freitas et al. 2012; Lima et al. 2014; Lima et al. 2016a; Oliveira e Barros 2018; Santos et al. 2018; Bastos et al. 2020; Santos 2020).

O estado do Piauí é detentor de rica flora, onde muitas espécies encontram-se em risco de extinção decorrente da extração desordenada dos recursos naturais e perda de habitats para áreas de agropecuária e aquicultura (Santos-Filho et al. 2016). A vegetação é fonte de insumos para a reprodução cultural, alimentar, medicinal e obtenção de renda para vários grupos populacionais piauienses (Oliveira e Barros 2018; Santos et al. 2018), havendo a necessidade de políticas públicas que resguardem a flora, o modo de vida, regulamentação da comercialização e identificação das espécies nativas que são vendidas para fins terapêuticos, bem como as finalidades de tratamento.

Em Parnaíba, Piauí, as plantas nativas comercializadas em mercados públicos servem como alimentícias, ritualísticas e medicinais, colaborando na renda mensal de permissionários. Para os usuários da medicina popular comercializadas em ambientes públicos, as plantas são geralmente o recurso primário de atenção à saúde, seja pela falta de condições de adquirirem medicamentos, ou pelo difícil acesso ao sistema de saúde público ou privado. Diante desse panorama, objetivouse identificar como e quais plantas nativas medicinais estão sendo comercializadas nos Mercados Públicos da cidade de Parnaíba, Piauí, e reconhecer as plantas mais versáteis, as partes utilizadas, forma de preparo e administração.

\section{Material e Métodos}

\section{Área de estudo}

Selecionou-se o município de Parnaíba para a presente pesquisa. Parnaíba está localizada na mesorregião norte do estado Piauí, microrregião do Litoral Piauiense ( $02^{\circ} 54$ `34,00"S; $41^{\circ} 47^{`} 13,00^{\prime}$ W), densidade demográfica de 334, 51 hab/ km² (IBGE 2016), com população estimada de 152.653 e detentor de quatro mercados públicos (Figura 1). O clima é caracterizado como Tropical Quente, com temperatura variando entre $25^{\circ} \mathrm{C}$ a $33^{\circ} \mathrm{C}$ (Weather Spark 2019). De acordo com Fernandes (1981) a vegetação do Piauí apresenta-se em: florestas, cocais (carnaubais, babaçuais e buritizais) carrasco, vegetação campestre e vegetação litorânea. Santos-Filho et al. (2009) caracterizaram a vegetação piauiense com áreas de transição entre caatinga, cerrado e restinga. 
Figura 1. Localização dos mercados Públicos em Parnaíba, Piauí, Brasil
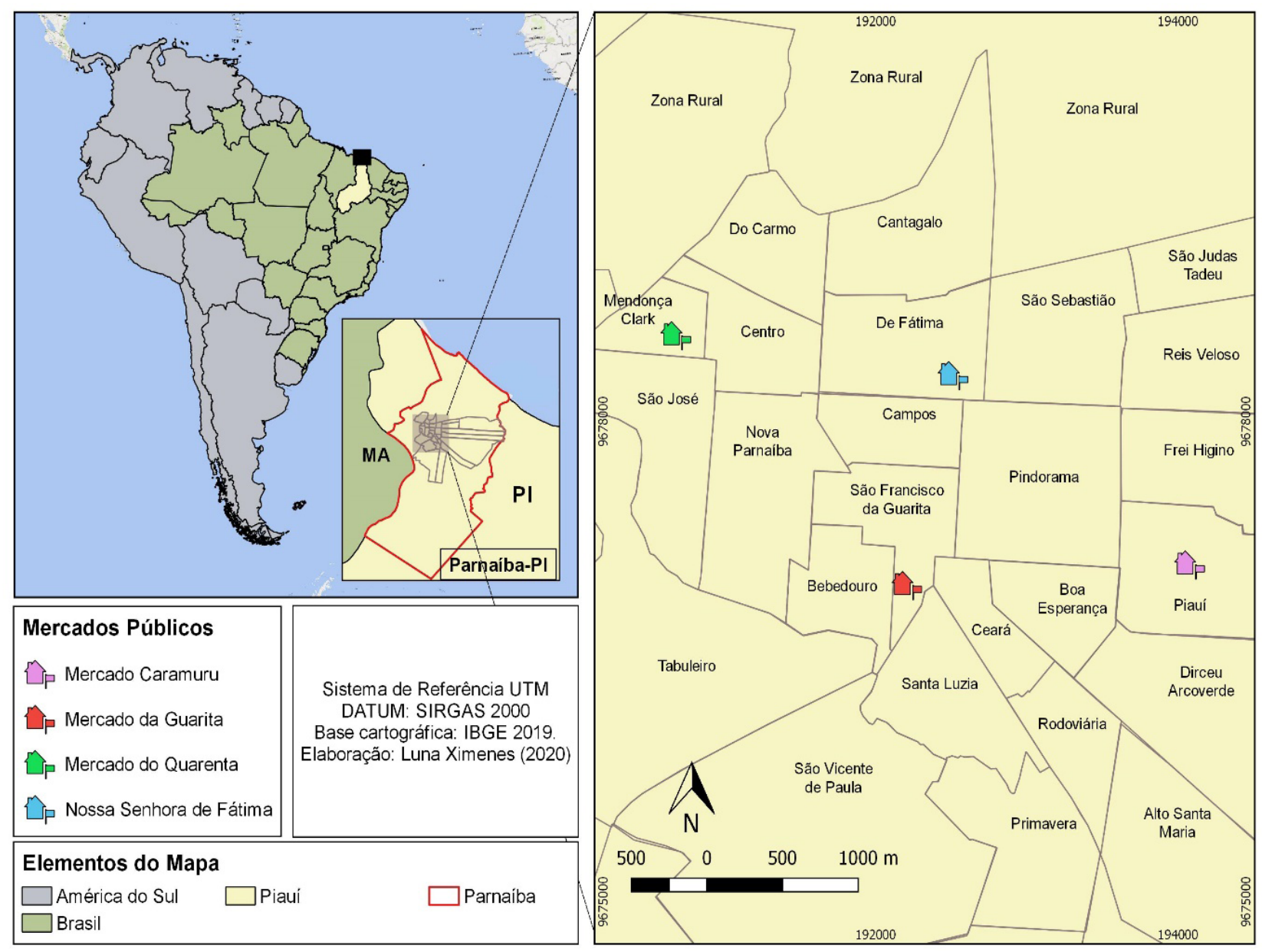

\section{Coleta de dados}

A pesquisa foi realizada nos meses de agosto de 2018 e maio de 2019. Em atendimento a Resolução 466/12 - CNS, a presente pesquisa foi aprovada pelo Comitê de Ética em Pesquisas (CEP) da Universidade Federal do Piauí (UFPI) sob o $\mathrm{N}^{\circ} 2.975 .850$, com cadastro no Sistema Nacional de Gestão do Patrimônio Genético e do Conhecimento Tradicional Associado (SISGEN), sob o N ${ }^{\circ}$ ABB2F8B.

Foram selecionados todos os quatro mercados públicos para a realização da investigação. A técnica de "rapport" (Barbosa 2007) foi utilizada na conquista da confiança dos vendedores de plantas medicinais e posteriormente realizadas entrevistas por meio de formulários padronizados (Bernard 1988) com todos os vendedores de ervas medicinais com idade superior a 18 anos. Foram questionados 34 informantes nos mercados da Quarenta (n:14), Caramuru (n: 11), Nossa Senhora de Fátima (n: 7), Guarita (n:2). Estes espaços de comercialização distam em média 2,14 km entre si (Figura 2). 
Figura 2. Mercados públicos municipais de Parnaíba, Piauí: A) Fátima; B) “Quarenta”; C) Caramuru; D) Guarita.

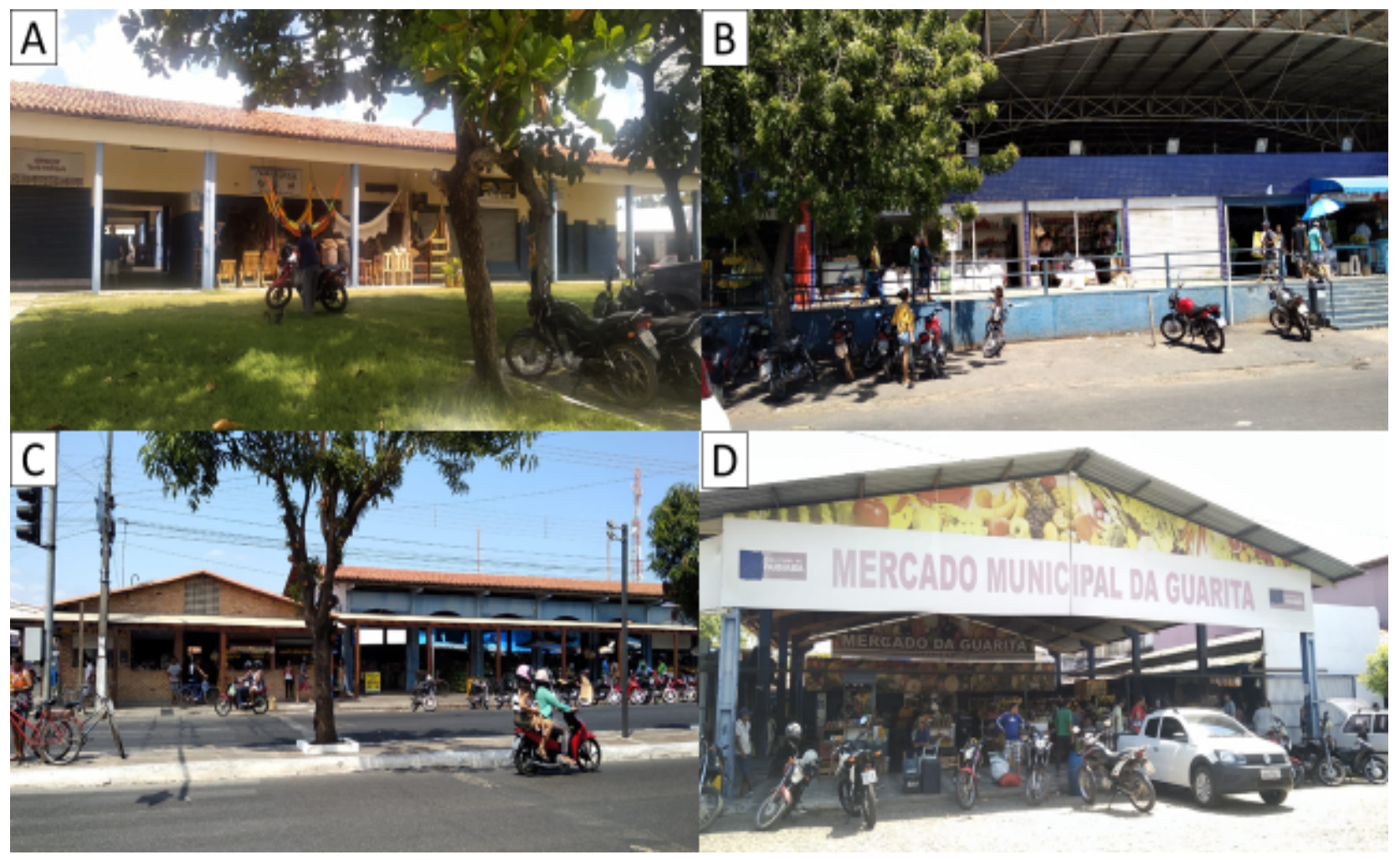

Os informantes foram questionados sobre as plantas comercializadas, modo de obtenção, formas de uso e preparo, finalidades de tratamento e demais questionamentos. Além disso, vídeos, fotos e áudios foram utilizados, com a permissão assinada no Termo de Consentimento Livre e Esclarecido (TCLE).

Plantas passíveis de identificação nos mercados públicos, foram herborizadas (Mori et al. 1989). As demais, em virtude a inviabilidade da realização da identificação no local, por se limitarem a partes vegetativas, foram coletadas por meio de "turnês-guiadas" (Bernard 1988) com dois erveiros. Estas plantas foram identificadas com auxílio de bibliografia ou por comparação com material incorporado nos Herbários Graziela Barroso (TEPB) da Universidade Federal do Piauí - UFPI e HDELTA da Universidade Federal do Delta do Parnaíba (UFDPar) e posteriormente incorporadas ao Herbário TEPB.

Os nomes científicos das plantas e a verificação das abreviações dos nomes dos autores foram realizadas por intermédio da base de dados do Missouri Botanical Garden (MOBOT 2019), classificadas de acordo com Angiosperm Phylogeny Group (APG IV 2016), agrupadas em categorias de enfermidades de acordo com a Organização Mundial de Saúde (OMS 2007), e a Flora do Brasil on line (2020) foi utilizada para a confirmação do status das espécies.

\section{Análise dos dados}

Avaliaram-se os dados coletados por intermédio do Fator do Consenso do Informante -FCI (Trotter e Logan 1986) usado para medir a importância das espécies mais frequetemente usadas, 
de acordo com os informantes. Foi verificado por meio da seguinte fórmula: FCI = Nur-Nt / Nur-1, onde Nur indica o número de citações de utilização em cada categoria e Nt o número de espécies usadas. O valor máximo do FCI é 1, e indica concordância entre os informantes sobre as espécies selecionadas para uma determinada categoria de uso.

Calculou-se a Importância Relativa (IR) das espécies nativas citadas por cada informante e as plantas que obtiveram os valores mais elevados (máximo $=2$ ) correspondem às apontadas para um maior número de sistemas corporais e consideradas as mais versáteis (Bennett e Prance 2000). A fórmula utilizada: IR = NSC + NP, onde IR é a importância relativa, NSC o número de sistemas corporais obtido pela razão entre o número de sistemas corporais tratados por uma determinada espécie (NSCE) e o número total de sistemas corporais tratados pela espécie mais versátil (NSCEV). O NP é a razão entre o número de propriedades atribuídas a uma determinada espécie (NPE) e o número total de propriedades impostas à espécie mais versátil (NPEV). Também foi utilizado o Índice de Similaridade de Jaccard que expressa a semelhança entre ambientes, baseando-se no número de espécies comuns (Magurran 1988 e Brower et al. 1997).

\section{Resultados e Discussão}

\section{Caracterização socioeconômica dos vendedores de plantas medicinais}

Em relação aos dados sobre o perfil dos entrevistados, verificou que 50\% eram homens e $50 \%$ mulheres. A equidade na participação dos gêneros é comumente registrada nestes espaços de comercialização (Lima et al. 2011; Alves et al. 2016; Santos et al. 2018). Isto sugere que nesta atividade há oportunidade igualitárias para ambos os sexos.

A maioria dos entrevistados (62\%) apresentou idade entre 25 a 59 anos; $32 \%$ com mais de 60 anos; 3\% nas faixas etárias de 18-24 anos e 3\% não relatou. Estes dados indicam a ascendência de adultos em mercados públicos. É frequente a predominância de adultos comerciantes em mercados brasileiros (Sousa et al. 2017; Santos et al. 2018) e internacionais (Giraldo et al. 2015). Poucos são os vendedores jovens de plantas no município de Parnaíba. Pressupõe-se que há uma preferência dos mais jovens em trabalhar em outras atividades comerciais diante das ofertas de emprego no município. A falta de entusiasmo das gerações mais novas com a venda de plantas poderá representar uma ameaça de perda de informações valiosas sobre os recursos vegetais da flora brasileira (Araújo et al. 2009).

A renda obtida com a venda de espécies medicinais nativas variou entre os informantes, no qual $15 \%$ recebem menos de um salário, $82 \%$ recebem mais de um salário e 3\% não relatou. O tempo de atuação comercial nos mercados variou de um a 40 anos, obtendo-se a média aproximada de 24 anos de trabalho neste ramo. É comum vendedores de plantas trabalhando em mercados públicos há mais de 10 anos (Lima et al. 2011; Ferreira, 2014; Santos et al. 2018), mas, diferentemente do registrado nessa pesquisa, predomina a baixa renda entre os erveiros de mercados brasileiros (Lima et al. 2016a). Isso reflete que a comercialização de plantas em mercados públicos desempenha importante contribuição na renda familiar, desestimulando a mudança de atividade.

Quanto à escolaridade, 6\% não são escolarizados, 64\% iniciou o ensino fundamental, 15\% possui o ensino fundamental completo, $3 \%$ iniciou o ensino médio, e $12 \%$ informou ter o ensino médio completo. A baixa escolaridade dos erveiros em mercados públicos foi salientada em outros 
estudos etnobotânicos (Araújo et al. 2009; Bastos et al. 2020). A escolaridade verificada, indica que a comercialização de plantas medicinais na cidade de Parnaíba é uma situação favorável para os vendedores que não possuem assistência escolar. Já que a venda de plantas não exige formação escolar específica.

A maioria dos comerciantes de plantas nasceram no Piauí (47,07\%), os demais são naturais do Ceará $(29,41 \%)$ e Maranhão (17,64\%). Os demais informaram a naturalidade em outros Estados. Geralmente, os indivíduos que trabalham em mercados públicos são naturais do próprio município que residem (Santos, 2018; Medeiros et al. 2019; Bastos et al. 2020). Indicando que os vendedores de plantas medicinais optam por trabalhar próximo do local de nascimento, pelo acesso facilitado na busca de plantas na natureza em detrimento do conhecimento adquirido ao longo dos anos naquela comunidade.

\section{Conhecimento sobre as plantas comercializadas}

Durante as entrevistas etnobotânicas em mercados públicos é normal verificar os vendedores de ervas consultando livros de plantas medicinais para certificarem os nomes das espécies, indicações e formas de usos (Ramos et al. 2005). A maioria dos comerciantes de plantas medicinais de Parnaíba (88\%) adquirem o conhecimento com os consumidores, mateiros, fornecedores e outros vendedores de plantas, além das consultas em livros de plantas medicinais e 12\% relataram adquirir conhecimento sobre as plantas a partir da convivência com os familiares (avós, pais, cônjuges e tios). Isto sugere que nos mercados parnaibanos há uma transmissão oral entre comerciantes e consumidores.

Vendedores brasileiros de plantas medicinais comumente adquirem as espécies vegetais com fornecedores, poucos cultivam ou coletam (Alves et al. 2016; Andrade et al. 2017). O mesmo foi observado nesta pesquisa, na qual $79 \%$ dos vendedores de plantas medicinais não coletam as plantas vendidas e sete (21\%) cultivam e coletam as plantas em hortas domésticas, quintais e matas próximas do município de Parnaíba, Piauí. Propõe-se que esta preferência seja decorrente da impossibilidade de sair do boxe de venda sem fechá-lo e a comodidade na compra com fornecedores que abastecem os negócios.

\section{Plantas comercializadas nos mercados públicos}

A partir das entrevistas foram registradas 46 espécies de plantas medicinais nativas, distribuídas em 29 famílias botânicas. As mais representativas em número de espécies, foram Fabaceae (10), Apocynaceae (3), Malvaceae (3), Rubiaceae (3), Anacardiaceae (2), Euphorbiaceae (2) e Meliaceae (2) (Tab. 1). É comum o uso de Fabaceae no tratamento de diversas enfermidades (Lima et al. 2011; Lima et al. 2014; Lima et al. 2016b; Santos et al. 2018). Esta família é a maior no Brasil, estando representada em todos os biomas brasileiros (BFG 2015) por sua abundância e riqueza na natureza (Santos et al. 2007), facilitando o acesso a essas espécies. 


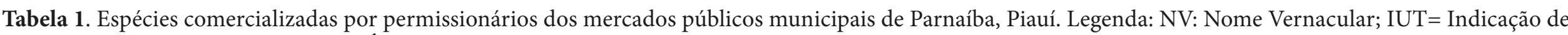

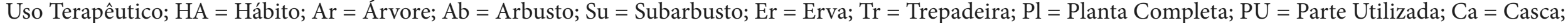

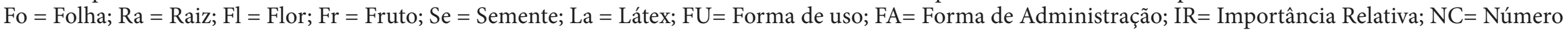
de Citações.

\begin{tabular}{|c|c|c|c|c|c|c|c|c|}
\hline Família/Espécie & NV & HÁ & PU & IUT & FU & FA & IR & $\mathrm{NC}$ \\
\hline \multicolumn{9}{|l|}{ Acanthaceae } \\
\hline Justicia pectoralis Jacq. & Anador & Er & Fo & $\begin{array}{l}\text { Gripe, Cefaleia, } \\
\text { Dor muscular }\end{array}$ & Infusão & $\begin{array}{l}\text { Ingerir, } \\
\text { inalar }\end{array}$ & 0,44 & 3 \\
\hline \multicolumn{9}{|l|}{ Anacardiaceae } \\
\hline Anacardium occidentale L. & Cajui & Ar & $\mathrm{Ca}$ & $\begin{array}{l}\text { Cicatrizante, } \\
\text { Inflamação pélvica }\end{array}$ & Decocção & Ingerir & 0,38 & 2 \\
\hline Myracrodruon urundeuva Allemão & Aroeira & Ar & $\mathrm{Ca}$ & $\begin{array}{l}\text { Inflamação pélvica, } \\
\text { Nefrite, Cicatrizante, } \\
\text { Próstatite, Fortificante, } \\
\text { Menopausa, Anemia }\end{array}$ & Garrafada & $\begin{array}{l}\text { Ingerir, } \\
\text { lavagem }\end{array}$ & 1,94 & 8 \\
\hline \multicolumn{9}{|l|}{ Apocynaceae } \\
\hline Aspidosperma pyrifolium Mart. \& Zucc. & Pereira & Ar & $\mathrm{Ca}$ & Diabetes & Decocção & Ingerir & 0,19 & 1 \\
\hline Hancornia speciosa Gomes & Mangaba & Ar & Fr & Pressão alta & In natura & Ingerir & 0,19 & 1 \\
\hline Himatanthus drasticus (Mart.) Plumel & Janaguba & Ar & Lá & $\begin{array}{l}\text { Anemia, Gastrite } \\
\text { Inflamação pélvica, } \\
\text { Depurativa }\end{array}$ & Garrafada & $\begin{array}{l}\text { Ingerir, } \\
\text { uso } \\
\text { tópico }\end{array}$ & 1,06 & 7 \\
\hline \multicolumn{9}{|l|}{ Arecaceae } \\
\hline Copernicia prunifera (Mill.) H. E. Moore & Carnaúba & Ar & $\mathrm{Ra}$ & $\begin{array}{l}\text { Sarna, Furúnculo, } \\
\text { Cervicite, Nódulos }\end{array}$ & Infusão, & Ingerir & 0,81 & 5 \\
\hline \multicolumn{9}{|l|}{ Asteraceae } \\
\hline Solidago chilensis Meyen & Arnica & Er & Fo & $\begin{array}{l}\text { Dores musculares, } \\
\text { Gripe, Bronquite }\end{array}$ & Infusão & Ingerir & 0,63 & 4 \\
\hline \multicolumn{9}{|l|}{ Bignoniaceae } \\
\hline Handroanthus impetiginosus (Mart.ex DC.) Mattos & $\begin{array}{l}\text { Podarco- } \\
\text { Roxo }\end{array}$ & $\mathrm{Ar}$ & $\mathrm{Ca}$ & $\begin{array}{l}\text { Anemia, Fortificante, } \\
\text { Coceira, Fraqueza }\end{array}$ & Garrafada & Ingerir & 0,69 & 5 \\
\hline
\end{tabular}




\begin{tabular}{|c|c|c|c|c|c|c|c|c|}
\hline Família/Espécie & NV & HÁ & PU & IUT & FU & FA & IR & NC \\
\hline \multicolumn{9}{|l|}{ Bixaceae } \\
\hline Bixa orellana L. & Urucum & $\mathrm{Ab}$ & $\mathrm{Se}$ & $\begin{array}{l}\text { Doença cardiovascular, } \\
\text { Acidente } \\
\text { Vascular Cerebral }\end{array}$ & Infusão & Ingerir & 0,25 & 2 \\
\hline \multicolumn{9}{|l|}{ Bromeliaceae } \\
\hline Ananas comosus (L.) Merr. & Abacaxi & Er & Fr & $\begin{array}{l}\text { Gripe, Faringite, } \\
\text { Tosse, Resfriado } \\
\text { Coriza, Asma }\end{array}$ & Decocção & Ingerir & 0,63 & 6 \\
\hline \multicolumn{9}{|l|}{ Caryocaraceae } \\
\hline Caryocar coriaceum Wittm. & Pequi & Ar & Fr & $\begin{array}{l}\text { Gripe, Doença } \\
\text { cardiovascular, } \\
\text { Má digestão }\end{array}$ & Óleo & Ingerir & 1,13 & 10 \\
\hline \multicolumn{9}{|l|}{ Cleomaceae } \\
\hline Tarenaya spinosa (Jacq.) Raf. & Mussambe & $\mathrm{Ab}$ & $\mathrm{Pl}$ & $\begin{array}{l}\text { Fortificante, Anemia } \\
\text { Gripe, Tosse, Asma }\end{array}$ & $\begin{array}{l}\text { Infusão, } \\
\text { Garrafada }\end{array}$ & Ingerir & 1,00 & 8 \\
\hline \multicolumn{9}{|l|}{ Combretaceae } \\
\hline Combretum leprosum Mart. & Mufumbo & $\mathrm{Ab}$ & Fo & $\begin{array}{l}\text { Hemorragia, Gripe, } \\
\text { Tosse, Cicatrizante, } \\
\text { Bronquite, Calmante } \\
\text { Asma, Má digestão }\end{array}$ & Decocção, garrafada & Ingerir & 0,94 & 9 \\
\hline \multicolumn{9}{|l|}{ Convolvulaceae } \\
\hline Operculina macrocarpa L. & Jalapa & $\operatorname{Tr}$ & $\mathrm{Ra}$ & $\begin{array}{l}\text { Acidente vascular } \\
\text { cerebral, } \\
\text { Dores musculares }\end{array}$ & Decocção, garrafada & $\begin{array}{l}\text { Ingerir, } \\
\text { uso } \\
\text { tópico }\end{array}$ & 0,75 & 6 \\
\hline \multicolumn{9}{|l|}{ Cucurbitaceae } \\
\hline Luffa operculata L. & Cabacinha & $\operatorname{Tr}$ & $\mathrm{Fr}$ & Sinusite, Abortiva & Decocção & $\begin{array}{l}\text { Ingerir, } \\
\text { inalar }\end{array}$ & 0,75 & 4 \\
\hline
\end{tabular}




\begin{tabular}{|c|c|c|c|c|c|c|c|c|}
\hline Família/Espécie & NV & HÁ & PU & IUT & FU & FA & IR & NC \\
\hline \multicolumn{9}{|l|}{ Dilleniaceae } \\
\hline Curatella americana $\mathrm{L}$. & Sambaiba & $\mathrm{Ab}$ & $\mathrm{Ra}$ & Gastrite, Anemia & Decocção, garrafada & Ingerir & 0,38 & 2 \\
\hline \multicolumn{9}{|l|}{ Euphorbiaceae } \\
\hline Croton sp & Mameleiro & $\mathrm{Ab}$ & $\mathrm{Ca}$ & $\begin{array}{l}\text { Dor de estômago, } \\
\text { Diarreia }\end{array}$ & Infusão, decocção & Ingerir & 0,50 & 4 \\
\hline Jatropha gossypiifolia L. & Pião-roxo & $\mathrm{Su}$ & $\mathrm{Pl}$ & Anti-inflamatório & Maceração & $\begin{array}{l}\text { Uso } \\
\text { tópico }\end{array}$ & 0,19 & 1 \\
\hline Amburana cearensis (Allemão.) A.C.Sm. & Emburana & $\mathrm{Ar}$ & $\mathrm{Ca}$ & $\begin{array}{l}\text { Dor de estômago, } \\
\text { Diarreia, Gripe }\end{array}$ & Garrafada & Ingerir & 0,56 & 3 \\
\hline Anadenanthera peregrina (L.) Speg. & Angico & $\mathrm{Ab}$ & $\mathrm{Ca}$ & $\begin{array}{l}\text { Contusão, Faringite, } \\
\text { Tosse, Gripe, Asma, } \\
\text { Pneumonia, Resfriado }\end{array}$ & Garrafada & $\begin{array}{l}\text { Ingerir, } \\
\text { gargarejo }\end{array}$ & 0,94 & 9 \\
\hline Bauhinia forficata Link & Pata-de-vaca & $\mathrm{Ar}$ & Fo & $\begin{array}{l}\text { Diabetes, Pancreatite, } \\
\text { Diarreia, Anemia, } \\
\text { Prisão-de-ventre }\end{array}$ & Decocção & Ingerir & 0,81 & 5 \\
\hline Copaifera sp. & Copaíba & Ar & $\mathrm{Ca}, \mathrm{Fr}$ & $\begin{array}{l}\text { Anti-inflamatório, } \\
\text { Cicatrizante }\end{array}$ & Óleo & $\begin{array}{l}\text { Uso } \\
\text { tópico }\end{array}$ & 1,88 & 16 \\
\hline $\begin{array}{l}\text { Hymenaea stigonocarpa } \\
\text { Mart. ex Hayne }\end{array}$ & Jatobá & Ar & $\mathrm{Ca}, \mathrm{Fr}$ & $\begin{array}{l}\text { Gripe, Pneumonia, } \\
\text { Resfriado, Prostatite, } \\
\text { Faringite, Asma, } \\
\text { Anti-inflamatório }\end{array}$ & Decocção & Ingerir & 1,13 & 10 \\
\hline Libidibia ferrea (Mart. ex Tul.) L. P.Queiroz & Jucá & Ar & $\mathrm{Se}$ & $\begin{array}{l}\text { Gastrite, Gripe, Nefrite } \\
\text { Dor de estômago, } \\
\text { Infecção urinária, } \\
\text { Cicatrizante, Diabetes } \\
\text { Prostatite, Artrose }\end{array}$ & Decocção, garrafada & $\begin{array}{l}\text { Ingerir, } \\
\text { lavagem }\end{array}$ & 1,94 & 15 \\
\hline
\end{tabular}




\begin{tabular}{|c|c|c|c|c|c|c|c|c|}
\hline Família/Espécie & NV & HÁ & PU & IUT & FU & FA & IR & NC \\
\hline Mimosa caesalpiniifolia Benth. & Unha-de-gato & $\mathrm{Ab}$ & Fo & $\begin{array}{l}\text { Gripe, Pressão-alta, } \\
\text { Reumatismo, Tosse, } \\
\text { Inflamação Pélvica, } \\
\text { Bronquite, Artrose }\end{array}$ & Infusão & Ingerir & 1,56 & 11 \\
\hline Mucuna pruriens (L.) DC. & Mucuná & $\operatorname{Tr}$ & $\mathrm{Se}$ & Hemorroidas & In natura & $\begin{array}{l}\text { Uso } \\
\text { tópico }\end{array}$ & 0,19 & 1 \\
\hline $\begin{array}{l}\text { Stryphnodendron adstringens (Mart.) } \\
\text { Coville }\end{array}$ & Barbatimão & $\mathrm{Ab}$ & $\mathrm{Fo}, \mathrm{Ca}$ & $\begin{array}{l}\text { Inflamação pélvica, } \\
\text { Anti-inflamatório, } \\
\text { Cicatrizante, Mioma }\end{array}$ & Garrafada & $\begin{array}{l}\text { Ingerir, } \\
\text { lavagem }\end{array}$ & 0,50 & 4 \\
\hline \multicolumn{9}{|l|}{ Iridaceae } \\
\hline Eleutherine bulbosa (Mill.) Urb. & Palmerinha & Er & $\mathrm{Bu}$ & Diarreia & Decocção & Ingerir & 0,19 & 1 \\
\hline \multicolumn{9}{|l|}{ Lecythidaceae } \\
\hline Eschweilera sp & Imbiriba & Ar & $\mathrm{Se}$ & $\begin{array}{l}\text { Inflamação pélvica, } \\
\text { Fraqueza, Gastrite, } \\
\text { Diarreia, Cefaleia, } \\
\text { Anti-inflamatório }\end{array}$ & Garrafada & $\begin{array}{l}\text { Ingerir, } \\
\text { lavagem }\end{array}$ & 1,69 & 15 \\
\hline \multicolumn{9}{|l|}{ Malvaceae } \\
\hline Ceiba sp & Barriguda & Ar & $\mathrm{Ca}$ & $\begin{array}{l}\text { Anti-inflamatório, } \\
\text { Hérnia, Prostatite, } \\
\text { Îngua, Artrose }\end{array}$ & Garrafada & Ingerir & 0,81 & 5 \\
\hline Hibiscus sabdariffa L. & Hibiscus & $\mathrm{Ab}$ & Fo,Fl & $\begin{array}{l}\text { Anti-inflamatório, } \\
\text { Artrite }\end{array}$ & Infusão, decocção & Ingerir & 0,69 & 5 \\
\hline Luehea candicans Mart. \& Zucc. & Açoita-cavalo & Ar & $\mathrm{Ca}$ & $\begin{array}{l}\text { Anemia, Gastrite, } \\
\text { Anti-inflamatório, } \\
\text { Prostatite, Hérnia, } \\
\text { Esteatose hepática, } \\
\text { Nefrite }\end{array}$ & Decocção, garrafada & Ingerir & 1,19 & 7 \\
\hline \multicolumn{9}{|l|}{ Meliaceae } \\
\hline Carapa guianensis Aubl. & Andiroba & Ar & $\mathrm{Se}$ & $\begin{array}{l}\text { Mancha na pele, } \\
\text { Anti-inflamatório, } \\
\text { Cicatrizante, Otalgia }\end{array}$ & Óleo & $\begin{array}{l}\text { Ingerir, } \\
\text { Uso } \\
\text { tópico }\end{array}$ & 0,69 & 5 \\
\hline
\end{tabular}




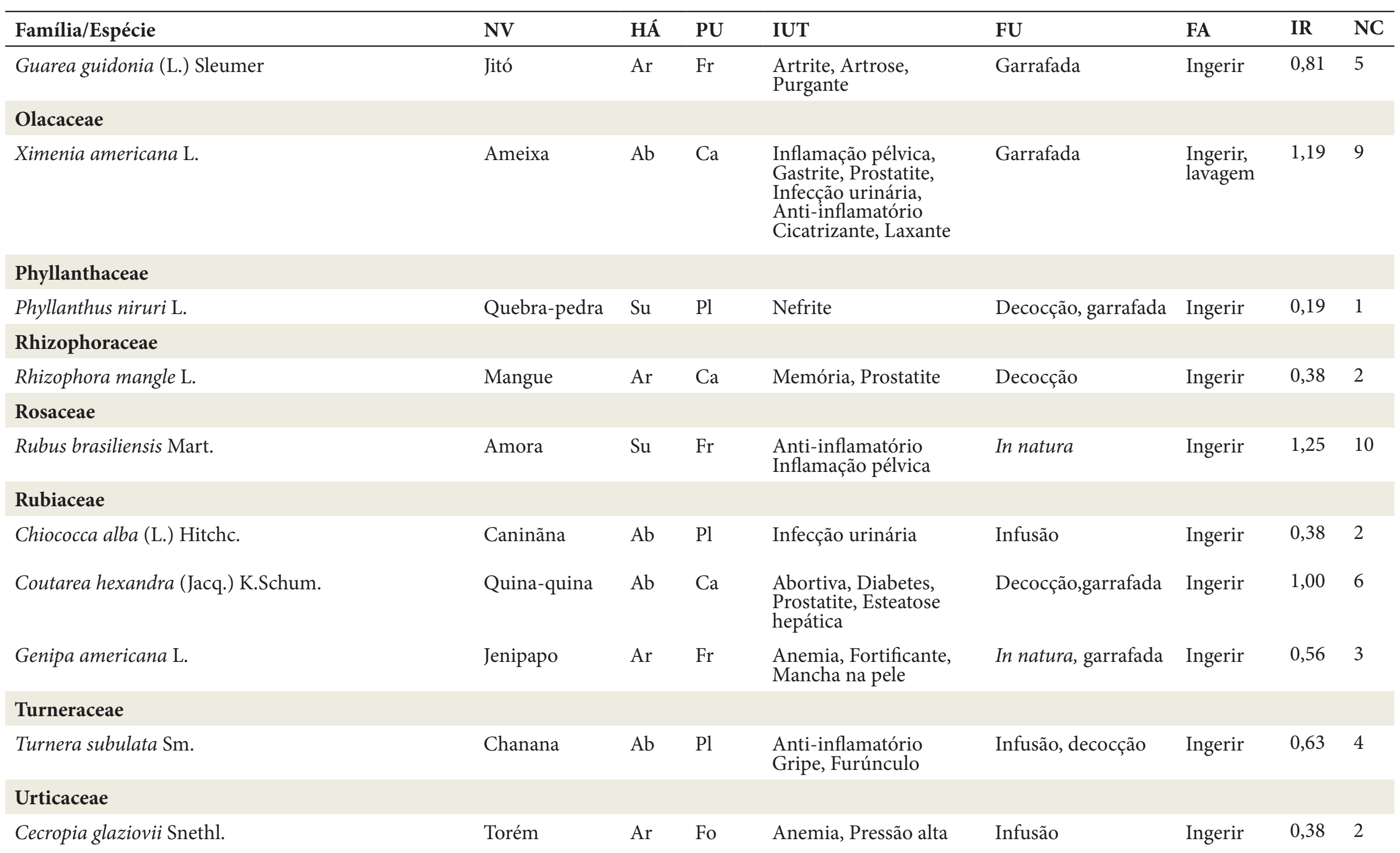




\begin{tabular}{|c|c|c|c|c|c|c|c|c|}
\hline Família/Espécie & NV & HÁ & PU & IUT & FU & FA & IR & NC \\
\hline \multicolumn{9}{|l|}{ Verbenaceae } \\
\hline Lippia alba (Mill.) N.E.Br. ex P. Wilson & Erva-cidreira & $\mathrm{Su}$ & Fo & $\begin{array}{l}\text { Calmante, Pressão alta, } \\
\text { Insônia, Febre }\end{array}$ & Infusão & Ingerir & 0,69 & 5 \\
\hline
\end{tabular}

Observou-se que 88,23\% das espécies são nativas e 11,77\% exóticas. É comum a sobreposição do uso das espécies nativas sobre as exóticas (Macêdo et al. 2015; Santos et al. 2018; Santos et al. 2019). Isso reflete o uso das plantas na cultura local, a forma de transmissão de conhecimento provinda dos antepassados e a facilidade de aquisição das plantas nativas.

O mercado da "Quarenta" destaca-se como núcleo diversificado de produtos medicinais e apresenta o maior número de ocorrências exclusivas de espécies (nove): mufumbo (C. leprosum Mart.), carnaúba (C. prunifera (Mill.) H. E. Moore.), cajuí (A. occidentale L.), chanana (T. subulata Sm.), mucuna (M. pruriens (L.) DC.), mangaba (H. speciosa Gomes.), pereira (A. pyrifolium Mart. \& Zucc.), sambaíba (C. americana L.), e torém (C. glaziovii Snethl) (Figura 3).

Figura 3. Dendograma de similaridade de Jaccard das espécies medicinais comercializadas nos mercados públicos de Parnaíba, Piauí.

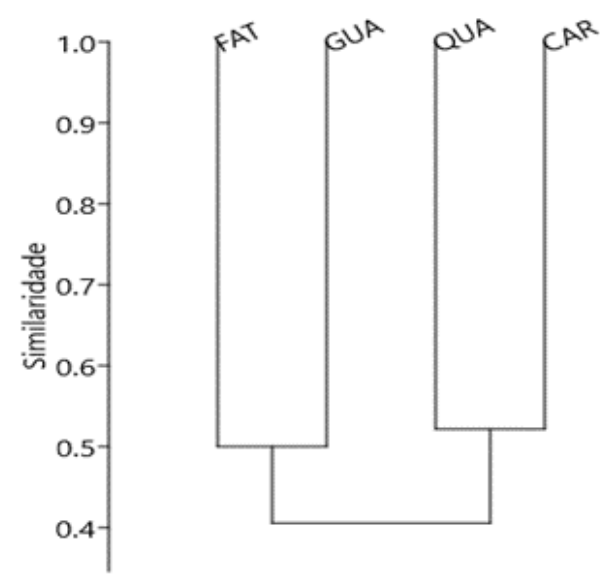


Parte da similaridade do mercado da "Quarenta" com o mercado da Caramuru é decorrente da afinidade dos erveiros com os fornecedores dos produtos que abastecem ambos os locais. Nesse contexto, algumas das espécies medicinais nativas, informadas neste estudo, são geralmente documentadas em outros estudos etnobotânicos em mercados públicos e feiras livres, como o $C$. leprosum Mart., e Copaifera sp., (Lima et al. 2016a; Oliveira e Barros 2018; Santos et al. 2018).

Vale ressaltar que a A. cearenses (Allemão.) A. C. Sm., M. urundeuva Allemão., L. candicans Mart. \& Zucc, estão presentes na Lista Oficial das Espécies da Flora Brasileira Ameaçadas de Extinção (Livro vermelho 2013) e as espécies B. nitida Spruce ex Benth., H. impetiginosus (Mart.ex DC.) Mattos e C. americana L., são as espécies madeireiras mais comercializadas do Brasil e apresentam interesse comercial, devido a madeira de boa qualidade (Sistema Nacional de Informações Florestais 2019). Isto indica a necessidade de estudos futuros avaliando como as vertentes dessa comercialização afeta a conservação da flora piauiense.

Apesar das espécies herbáceas destacarem-se no litoral do Piauí (Santos-Filho et al. 2016), o hábito arbóreo $(62,50 \%)$ e arbustivo $(14,56 \%)$ são os mais presentes na comercialização, seguido de subarbustivo (10,46\%), lianescente $(6,24 \%)$ e herbáceo $(6,24 \%)$. Este padrão se repete em outros mercados brasileiros (Almeida e Albuquerque 2002; Lima et al. 2011; Santos et al. 2018). Provavelmente este padrão esteja vinculado a facilidade de encontrar plantas com portes maiores na vegetação, contribuindo para a preferência deste hábito.

As espécies Copaifera sp., Eschweilera sp e Libidibia ferrea (Mart. ex Tul.) L. P. Queiroz., foram as mais citadas na presente pesquisa. As plantas nativas L. ferrea (jucá) e Myracrodruon urundeuva Allemão (aroeira), apresentaram maior diversidade de uso medicinal $(\mathrm{IR}=1,94)$. Estas espécies também foram registradas nas pesquisas etnobotânicas de Lima et al. (2014), Macêdo et al. (2015), Alves et al. (2016), Andrade et al. (2017), Santos et al. (2018) e Santos et al. (2019). O jucá (L. ferrea), alcançou o valor máximo de importância relativa entre as espécies citadas como medicinais, sendo a mais versátil, com o maior número de propriedades terapêuticas (nove). Além de suas atividades analgésica, anti-histamínica, antimicrobiana, anticoagulante e cicatrizante (Barakat 2011; Marreiro et al. 2014).

A M. urundeuva apresentou grande versatilidade de uso. Neste estudo a planta foi citada para tratar doenças do aparelho geniturinário (inflamação pélvica, prostatite), doenças endócrinas (menopausa), e doenças do sangue (anemia). A espécie é mencionada por Lucena et al. (2011) para o tratamento de inflamação do útero, inflamações intestinais, ferimentos externos, e infecções em gerais. Esta foi a mais citada por Lima et al. (2014) em feiras livres e mercados públicos de São Luís.

Dentre as estruturas vegetativas, observa-se uma predileção das cascas no tratamento popular de doenças no Nordeste brasileiro (Albuquerque et al. 2008; Lucena et al. 2008). Assim como no litoral piauiense. A parte da planta mais comercializada pelos erveiros são as cascas (37,22\%), seguida de folha $(20,16 \%)$, fruto $(16,23 \%)$, semente $(9,72 \%)$, planta inteira $(7,18 \%)$, raiz $(4,39 \%)$, flor $(2,55)$ e látex (2,55\%). Isso revela que o uso das cascas esteja vinculado a disponibilidade desta ao longo de todo o ano, ao contrário das demais estruturas vegetais que tendem à senescência durante o período de estiagem (Conceição et al. 2011; Lima et al. 2011; Lima et al. 2014). Os resultados advertem também para a necessidade de estudos que avaliem a retirada sem planejamento e manejo das matas locais.

As estruturas vegetais são utilizadas principalmente na forma de chás (38\%: decocção - 21\% e infusão $17 \%)$, seguido por garrafadas (29\%), in natura (16\%), óleo (12\%), e maceração (5\%). 
Em relação à forma de administração, a ingestão oral destacou-se $(52,23 \%)$, em seguida a lavagem (27,22\%), uso tópico (11,73\%), inalação $(5,88 \%)$, e gargarejo (2,94\%). É comum o uso dos chás e a ingestão de líquidos de forma oral ser a forma principal de administração (Santos et al. 2018; Barreto e Spanholi, 2019). Sugere-se que a escolha de chás, garrafadas e in natura esteja associada a facilidade de obtenção dos princípios ativos, diferentemente do processo de óleo e maceração que exigem técnica e tempo.

\section{Tratamento de doenças}

Considerando os sistemas corporais (Tabela 2), observa-se que houve concordância significativa entre os vendedores, no que se refere às indicações de uso para o tratamento de Distúrbios na gestação/outras doenças da mãe $(\mathrm{FCI}=1)$, Sintomas e sinais em gerais $(\mathrm{FCI}=0,59)$ e Doenças do sistema respiratório $(\mathrm{FCI}=0,56)$. Doenças do aparelho geniturinário recebeu 28 citações para 15 espécies. Destas, oito indicações foram para inflamação pélvica tendo M. urundeuva apresentado o maior consenso para este fim. A espécie mais relatada dentro da categoria Doenças do aparelho digestivo foi L. ferrea. Já as Doenças de pele/tecido celular subcutâneo e Doenças do sangue obtiveram FCI baixo, indicando que o número de citações foi aproximadamente igual ao número de espécies informadas. As enfermidades relatadas para estes sistemas foram: doenças de pele (mancha na pele, furúnculo) doenças do sangue (anemia), apresentando a garrafada do fruto do jenipapo ( $G$. americana) indicada no tratamento de ambas categorias. Neoplasias, leucemia, linfoma, Doenças endócrinas, nutricionais, metabólicas, Transtornos dos olhos e ouvidos tiveram o valor de FCI igual a zero, significando que não houve concordância quanto ao uso de nenhuma planta comercializada nos mercados.

Tabela 2. Consenso dos informantes para o tratamento de doenças pelas plantas comercializadas em mercados públicos do Município de Parnaíba, Piauí. NUR= Número de citações; NT = Número de espécies; FCI $=$ Fator do Consenso do informante.

\begin{tabular}{lccc}
\hline Categorias de doenças & NUR & NT & FCI \\
\hline Distúrbios na gestação/Outras Doenças da Mãe & 2 & 1 & 1 \\
Sintomas e sinais em gerais & 95 & 39 & 0,59 \\
Doenças do aparelho respiratório & 44 & 20 & 0,56 \\
Poliartropatias inflamatórias/dorsalgia & 25 & 13 & 0,5 \\
Doenças infecciosas parasitárias & 3 & 2 & 0,5 \\
Doenças do aparelho geniturinário & 28 & 15 & 0,48 \\
Doenças do aparelho digestivo & 32 & 18 & 0,45 \\
Doenças do aparelho circulatório & 20 & 14 & 0,31 \\
$\begin{array}{l}\text { Lesões, envenenamento e algumas outras consequências de causas } \\
\text { externas }\end{array}$ & 05 & 04 & 0,25
\end{tabular}




\begin{tabular}{lccc}
\hline Categorias de doenças & NUR & NT & FCI \\
\hline $\begin{array}{l}\text { Doenças de pele e do tecido subcutâneo: dermatite, unhas } \\
\text { Doenças do sangue e dos órgãos hematopoéticos e alguns transtornos }\end{array}$ & 06 & 05 & 0,2 \\
$\begin{array}{l}\text { imunitários } \\
\text { Neoplasias, leucemia linfoma }\end{array}$ & 13 & 12 & 0,08 \\
Doenças endócrinas, nutricionais, metabólicas & 9 & 9 & 0 \\
Transtornos dos olhos e ouvidos & 10 & 1 & 0 \\
\hline
\end{tabular}

$\mathrm{Na}$ categoria de doença Sintomas e sinais em gerais, obteve-se maiores indicações, devido à ampla procura por remédios anti-inflamatórios. Presume-se que as doenças respiratórias tiveram um número grande de citações e indicações terapêuticas, em virtude de ser uma das enfermidades mais frequente no cotidiano do parnaibano, já que no período chuvoso geralmente existe um aumento de gripes, resfriados e entre outras doenças. Estudos com plantas medicinais realizados em mercados públicos destacaram esse sistema corporal com maiores valores de citações (Almeida e Albuquerque 2002; Pilla et al. 2006).

\section{Conclusão}

A comercialização de plantas nativas em mercados públicos no município de Parnaíba é realizada por erveiros que precisam de fornecedores para abastecer seus estabelecimentos. Diversas espécies são empregadas no tratamento de doenças, principalmente as que mais assolam a população. Essa atividade representa fonte de renda para os vendedores e surge como uma possibilidade para trabalhadores informais que possuem conhecimento sobre a flora nativa em virtude da experiência diária com clientes, fornecedores e outros erveiros. Algumas plantas nativas medicinais estão em situação de risco. Deste modo, são necessários estudos futuros avaliando o impacto da coleta e de como esse comércio e a quantidade de usos impactam a flora regional.

\section{Agradecimentos}

Aos permissionários dos Mercados Públicos de Parnaíba, Piauí, que contribuíram para estes resultados e à Coordenação de Aperfeiçoamento de Pessoal de Nível Superior (CAPES), pela concessão da bolsa de estudo a primeira autora.

Participação dos autores: MHBS - Coleta de dados no Mercado da Quarenta e Mercado de Fátima, redação do artigo; JCF - Coleta de dados no Mercado da Caramuru e auxílio nas análises; BEM - Coleta de dados da Guarita e auxílio nas análises; IRV - Co-orientadora, análise dos dados e correção do artigo; RFMB - Orientador e supervisor da execução do projeto.

Aprovação ética: termos e o projeto foram cadastrados no Comitê de Ética em Pesquisas (CEP) da Universidade Federal do Piauí (UFPI) sob o N².975.850 e no Sistema Nacional de Gestão do Patrimônio Genético e do Conhecimento Tradicional Associado (SISGEN) em 02/10/2018, usuário da Universidade Federal do Piauí. 
Disponibilidade dos dados: Manuscrito desenvolvido a partir da dissertação de mestrado da primeira autora. Os dados estão disponíveis no Repositório Institucional da Universidade Federal do Piauí- (UFPI/PRODEMA) pelo link: https:// www.ufpi.br/repositorio-ufpi

Fomento: Coordenação de Aperfeiçoamento de Pessoal de Nível Superio - CAPES.

Conflito de interesses: os autores declaram não haver conflito de interesses.

\section{Referências}

Albuquerque UP, Monteiro JM, Ramos MA, Amorim ELC. 2007. Medicinal and magic plants from a public market in northeastern Brazil. Journal of Ethnopharmacology, v. 110, p. 76-91. DOI: https://doi.org/10.1016/j.jep.2006.09.010.

Albuquerque UP, Lucena RFP, Alencar NL. 2008. Métodos e técnicas para coleta de dados etnobotânicos. In: Albuquerque UP, Lucena RFP, Cunha LVFC. (Orgs.). Métodos e técnicas na pesquisa etnobotânica. COMUNIGRAF, Recife, p. 4172.

Almeida CFCB, Albuquerque UP. 2002. Uso e conservação de plantas e animais medicinais no estado de Pernambuco (Nordeste do Brasil): um estudo de caso. Interciência, v. 26, n. 6, p. 276-285.

Andrade LHC, Silva MCG, Trigueiros LMBM. 2017 Liquens em cascas de plantas medicinais comercializadas em mercados públicos da cidade do Recife, PE, Nordeste do Brasil. Arrudea, v. 3, n. 3, p. 1-41.

Alves, CAB, Silva S, Belarmino NALA, Souza RS, Silva DR, Alves PRR, Nunes GM. 2016. Comercialização de plantas medicinais: um estudo etnobotânico na feira livre do município de Guarabira, Paraíba, Nordeste do Brasil. Gaia Scientia, v. 10 , n. 4 , p. $390-407$.

APG (Angiosperm Phylogeny Group). 2016. An update of the angiosperm phylogeny group classification for the orders and families of flowering plants: APG IV. Botanical Journal of the Linnean Society, p. 181:1-20.

Araujo AC, Silva JP, Cunha JLXL, Araujo JLO. 2009. Caracterização socioeconômico-cultural de raizeiros e procedimentos pós-colheita de plantas medicinais comercializadas em Maceió, AL. Revista Brasileira de Plantas Medicinais, v. 11, n. 1, p. $81-91$.

Barbosa AR. 2007. Os humanos e os répteis da mata: uma abordagem etnoecológica de São José da Mata-Paraíba. 123 f. Dissertação (Mestrado em Desenvolvimento e Meio Ambiente). Universidade Federal da Paraíba/ João Pessoa.

Barakat L. 2011. Avaliação da viabilidade de obtenção de formas farmacêuticas plásticas contendo extrato seco de Caesalpinia ferrea Mart. 100 f. Dissertação (Mestrado em Ciências Farmacêuticas) - Universidade Federal do Amazonas, Manaus.

Barreto MR, Spanholi ML. 2019. Estudo etnobotânico em comunidades rurais de Sinop, Mato Grosso, Brasil. Interações, v. 20, n. 1, p. 1-1.

Bennett BC, Prance GT. 2000. Introduced plants in the indigenous pharmacopoeia of Northern South America. Economic Botany, v. 54, n. 1, p. 90-102.

Bernard HR. 1988. Research methods in cultural anthropology. Sage. Newbury Park, U.S.A, 520p.

BFG. 2015. Growing knowledge: an overview of Seed Plant diversity in Brazil. Rodriguésia, v. 66, p. 1085-1113.

Brower JE, Zar JH, Van Ende CN. 1997. Field and laboratory methods for general ecology. 4 th WCB/McGraw, New York. 273p.

Conceição GM, Ruggieri AC, Araújo MFV, Conceição TTMM, Conceição MAMM. 2011. Plantas do cerrado: comercialização, uso e indicação terapêutica fornecida pelos raizeiros e vendedores, Teresina, Piauí. Scientia Plena, v. 7 , n. 12, p. 1-6. 
Fernandes AG. 1981. Vegetação do Piauí. In: Livro de Anais do XXXII Congresso Nacional de Botânica. Teresina - PI: Editora da UFPI / Sociedade Botânica do Brasil, p. 7-9.

Ferreira JM. 2014. Plantas de uso medicinal e ritualístico comercializadas em mercados e feiras no Norte do Espírito Santo, Brasil. 92 f. Dissertação (Mestrado em Biodiversidade Tropical). Universidade Federal do Espírito Santo.

Freitas AVL, Azevedo RAB, Maia SSS. 2012. Os raizeiros e a comercialização de plantas medicinais em São Miguel, Rio Grande do Norte, Brasil, Revista Brasileira de Biociências, v. 10, n. 2, p.147-156.

Giraldo SEMQ, Lizarazú MCB, Robayo AM, Lobo AZP, Molano LG. 2015. Descrição do uso tradicional de plantas medicinais em mercados populares de Bogotá, D. C. Nova, v. 13, n. 23, p. 73-80.

Gomes NS, Lima JPS. 2017. Uso e comercialização de plantas medicinais em Humaitá, Amazonas, Revista Brasileira de Agroecologia, v. 12, n. 1, p. 019-031.

Hoang SV, Tran VC, D NTT. 2019. Traditional knowledge of local people on medicinal plants in Pu Hu nature reserve, Vietnam. Journal of Bioscience Discovery, v. 10, p. 72-102.

IBGE - Instituto Brasileiro de Geografia e Estatística. 2016. Censo demográfico, densidade demográfica, área territorial brasileira. Disponível em: https://cidades.ibge.gov.br/brasil/pi/parnaiba/pesquisa/30/30051. Acesso em: 20/03/2019.

Lima IEO, Nascimento LAM, Silva MS. 2016a. Comercialização de Plantas Medicinais no Município de Arapiraca-AL. Revista Brasileira de Plantas Medicinais, v. 18, n. 2, p. 462-472.

Lima PGC, Coelho-Ferreira M, Santos RS. 2014. A floresta na feira: plantas do município de Itaituba, Pará, Brasil. Fragmentos de cultura, v. 24, n. 2, p. 285-301.

Lima PGC, Coelho-Ferreira M, Oliveira R. 2011. Plantas medicinais em feiras e mercados públicos do Distrito Florestal Sustentável da BR-163, estado do Pará, Brasil. Acta Botanica Brasilica, v. 25, n. 2, p. 422-434.

Lima PGC, Coelho-Ferreira M, Santos RS. 2016b. Perspectives on medicinal plants in public markets across the Amazon: a review. Economic Botany, v. 70, n. 1, p. 64-78.

Linhares JFP, Hortegal EV, Rodrigues MIA, Silva PSS. 2014. Etnobotânica das principais plantas medicinais comercializadas em feiras e mercados de São Luís, Estado do Maranhão, Brasil. Revista Pan-Amazônica Saúde, v. 5, n. 3, p. 39-46.

Lucena RFP, Farias DC, Carvalho TKN, Lucena CM, Vasconcelos-Neto CFA, Albuquerque UP. 2011. Uso e conhecimento da aroeira (Myracrodruon urundeuva) por comunidades tradicionais no Semiárido brasileiro. Sitientibus série Ciências Biológicas, v. 11, n. 2, p. 255-264.

Lucena RFP, Nascimento VT, Araújo EL, Albuquerque UP. 2008. Local Uses of Native Plants in a Area of Caatinga Vegetation (Pernambuco, NE Brazil). Ethnobotanical Research \& Applications, v. 6, p. 03-13.

Macêdo DG, Ribeiro DA, Coutinho HDM, Menezes IRA, Souza MMA. 2015. Práticas terapêuticas tradicionais: uso e conhecimento de plantas do cerrado no estado de Pernambuco (Nordeste do Brasil). Boletín Latinoamericano y del Caribe de Plantas Medicinales y Aromáticas, v. 14, n. 6, p. 491-508.

Magurran AE. 1988. Ecological diversity and its measurement. Princeton University Press, New Jersey. 179p.

Marreiro RO, Bandeira MFCL, Souza, TPS, Almeida MC, Bendaham K. 2014. Evaluation of the stability and antimicrobial activity of an ethanolic extract of Libidibia ferrea. Clinical, Cosmetic and Investigational Dentistry, v. 6, p. 9-13.

Martinelli G, Moraes MA. 2013. (Org.). Livro vermelho da flora do Brasil. Instituto de Pesquisas Jardim Botânico do Rio de Janeiro, Rio de Janeiro, p. 1059-1060.

Medeiros FS, Braga de Sá G, Dantas MKL, Almeida MGVM. 2019. Survey of medicinal plants sold at the free trade fair of the municipality of Patos, Paraíba, Brazil. Revista Verde de Agroecologia e Desenvolvimento Sustentável, v. 14, n. 1, p. 150-155. 
MOBOT. Missouri Botanical Garden. 2018. Disponível em: http://www.missouribotanicalgarden.org/media/factpages/tropicos.aspx. Acesso em: 08/06/ 2018.

Mori AS, Silva LAM, Lisboa G, Coradin L. 1989. Manual de manejo do herbário fanerogâmico. CEPEC/CEPLAC, $104 \mathrm{p}$.

Oliveira FCS, Barros RFM. 2018. Aspectos sociodemográficos dos comerciantes de plantas medicinais na feira de Oeiras, Piauí. Revista Educação Ambiental em Ação, v. 63, p. 1-1.

Oliveira FCS, Barros RFM, Moita Neto JM. 2010. Plantas medicinais utilizadas em comunidades rurais de Oeiras, semiárido piauiense. Revista Brasileira de Plantas Medicinais, v. 12, n. 3, p. 282-301.

OMS - Organização Mundial de Saúde. 2007. Classificação Estatística Internacional de Doenças e Problemas Relacionados à Saúde. Décima Revisão, vol. I. Disponível em: http://www.who.int/classifications/icd/en. Acesso em: $22 / 07 / 2019$.

Pilla MAC, Amorozo MCM, Furlan A. 2006. Obtenção e uso das plantas medicinais no distrito de Martim Francisco, Município de Mogi-Mirim, SP, Brasil. Acta Botânica Brasílica, v. 20, n. 4, p. 1-10.

Ramos AR, Albuquerque UP, Amorim ELC. 2005. O comércio de plantas medicinais em mercados públicos e feiras livres: um estudo de caso. In: Albuquerque UP, Almeida CFCBR, Marins JFA. (Orgs.). Tópicos em conservação etnobotânica e etnofarmacológica de plantas medicinais e mágicas. NUPEEA. Recife: Universidade Federal Rural de Pernambuco, p. 127 - 163.

REFLORA - Flora do Brasil 2020. Algas, Fungos e Plantas. Disponível em: http://reflora.jbrj.gov.br/reflora/listaBrasil/ ConsultaPublicaUC/ConsultaPublicaUC.do\#CondicaoTaxonCP. Acesso em: 24/07/2019.

Roque AA, Rocha RM, Loiola MIB. 2010. Uso e diversidade de plantas medicinais da Caatinga na comunidade rural de Laginhas, município de Caicó, Rio Grande do Norte (nordeste do Brasil). Revista Brasileira de Plantas Medicinais, v. 12, n. 1, p. 31-42.

Santos-Filho FS. 2009. Composição florística e estrutural da vegetação de restinga do estado do Piauí. 120 f. Tese (Pós-Graduação em Botânica) Universidade Federal Rural de Pernambuco - UFRPE.

Santos-Filho FS, Mesquita TKS, Almeida Júnior EB, Zicke CS. 2016. A flora de Cajueiro da Praia: uma área de tabuleiros do litoral do Piauí, Brasil. Revista Equador, v. 5, n. 2, p. 21-35.

Santos MHB. 2020. Mercados públicos municipais de Parnaíba como difusores etnobotânicos no Piauí. $154 \mathrm{f}$. Dissertação (Mestrado em Desenvolvimento e Meio Ambiente). Universidade Federal do Piauí. Teresina.

Santos MV, Vieira IR, Silva MFS, Andrade IM. 2019. Comercialização de plantas medicinais nos mercados públicos do Município de Parnaíba, Piauí, Brasil. Revista Espacios, v. 40, n. 22, p. 1-1.

Santos RS, Lima PGC, Coelho-Ferreira MA. 2018. Etnobotânica de plantas medicinais em mercados públicos da Região Metropolitana de Belém do Pará, Brasil. Biota Amazônia, v. 8, n. 1, p. 1-9. DOI: http://dx.doi.org/10.18561/2179-5746/ biotaamazonia.v8n1p1-9.

Santos RM, Vieira FA, Fagundes M, Nunes YRF, Gusmão E. 2007. Riqueza e similaridade florística de oito remanescentes florestais no norte de Minas Gerais, Brasil. Revista Árvore, v. 31, n. 1, p. 135-144. DOI: http://dx.doi.org/10.1590/S010067622007000100015 .

SNIF - Sistema Nacional de Informações Florestais. 2019. Disponível em: http://snif.florestal.gov.br/pt-br/. Acesso em: 25/06/2019.

Silva SLC, Gualberto SA, Silva DC, Silveira TC, Macedo GEL. 2012. Plantas medicinais usadas pela comunidade do Povoado de Laços (Tanhaçú/Bahia) e encontradas na Floresta Nacional Contendas do Sincorá. Revista Caatinga (Online), v. 25, p. 130-136. 
Sousa E, Alves RJM, Silva JM, Dias NM, Silva LC. 2017. Socioeconomic prospection in open-airmarkets: the case of Vero-Peso Complex, Belém, Pará, Brazil. Revista Espacios, v. 38, n. 36, p. 1-5.

Trotter R, Logan M. 1986. Informant consensus: a new approach for identifying potentialli effective medicinal plants. In: ETKIN NL. Indigenous medicine and diet: behavioural approaches. 1 ed. New York, p. 91-112.

WEATHER SPARK - Condições meteorológicas médias de Parnaíba. Disponivel em: https://pt.weatherspark. com/y/30841/Clima-caracter\%C3\%ADstico-em-Parna\%C3\%ADba-Brasil-durante-o-ano. Acesso em: 22/03/2019. 